\title{
NUEVAS DINÁMICAS DE METROPOLIZACIÓN EN LAS REGIONES CONTEMPORÁNEAS. SINAPSIS TERRITORIAL DEL FRENTE FLUVIAL METROPOLITANO DE ROSARIO, ARGENTINA ${ }^{1}$
}

Artículo de Reflexión - Recibido 16 de Agosto de 2015 - Aceptado 15 de Noviembre de 2015

\author{
Cecilia Inés Galimberti
}

CURDIUR, FAPYD, UNR - CONICET. Rosario, Argentina. cecilia.galimberti@gmail.com

Para citar este artículo / to reference this article:

Galimberti, C. (2016). Nuevas Dinámicas de Metropolización en las Regiones Contemporáneas. Sinapsis territorial del frente fluvial metropolitano de Rosario, Argentina. Módulo Arquitectura CUC, Vol.16 №1, 9-34.

\begin{abstract}
Resumen
Desde las últimas décadas del siglo XX se registra una aceleración de procesos que se caracteriza por producir nuevas dinámicas de territorialización. Estas des-reterritorializaciones han cambiado las lógicas de los ámbitos metropolitanos, generando relaciones y flujos de interconexión que conducen hacia una nueva "metropolización". En este contexto, se considera que los límites taxativos de abordaje territorial resultan obsoletos y restrictivos. De manera que, tomando como caso de estudio la ribera metropolitana de Rosario -Argentina-, el presente artículo se propone demostrar las limitaciones del concepto tradicional de Área Metropolitana -AMR-, debido a las diversas dinámicas territoriales existentes, y se lo sustituye por el de Región Metropolitana. Se considera que la visión que plantea al Paraná como límite del AMR impide comprender las potencialidades y relaciones que suceden actualmente en este territorio. Por lo cual, se invierte este mirada, considerando al río como elemento estructurador de la misma.
\end{abstract}

\section{Palabras clave}

Territorialización, metropolización, frente fluvial, Región Metropolitana de Rosario.

\footnotetext{
1 El presente artículo se desprende de la tesis doctoral denominada "La reinvención del río. Procesos de transformación en la ribera de la Región Metropolitana de Rosario, Argentina".

2 Doctora en Arquitectura (2015) y Arquitecta (2008). CURDIUR, FAPyD, UNR - CONICET. Rosario, Argentina. cecilia. galimberti@gmail.com
} 


\section{NEW DYNAMICS OF METROPOLISATION IN THE CONTEMPORARY REGIONS. TERRITORIAL SYNAPSIS OF THE METROPOLITAN FLUVIAL FRONT OF ROSARIO, ARGENTINA}

\section{Abstract}

Since the last decades of the twentieth century is registered an acceleration of processes characterized by generating new dynamics of territorialisation. These des-reterritorialisations change the logics of the metropolitan areas, creating relationships and interconnection flows leading to a new "metropolisation". In this context, it is considered that taxative territorial limits turn out to be obsolete and restrictive. So, taking as a case of study the metropolitan banks of Rosario, the present article proposes to demonstrate the obsolescence of the traditional concept of Metropolitan Area - AMR-, given the diverse territorial dynamics that take place in recent decades, and it is replaced with the concept of Metropolitan Region. It is considered that the vision that raises the Paraná as limit of the AMR prevents from understanding the potentials and relations that happen nowadays in this territory. For which, this look is reversed, considering the river as a structural element of it.

\section{Keywords}

Territorialisation, metropolisation, waterfront, Metropolitan Region of Rosario. 


\section{Introducción}

Como plantean diversos autores (Harvey, 2000; Soja, 2008; Magnaghi, 2011), desde las últimas décadas del siglo XX se identifican cambios en los procesos de reestructuración territorial, identificándose una aceleración de los procesos de desterritorialización-reterritorialización. Por un lado, el primer término comprende una ruptura de los modos de producción tradicionales y de los patrones ligados a la identidad cultural y espacial en todas las escalas. Mientras que, por el otro lado, el segundo resulta una respuesta a estas reestructuraciones y requiere un nuevo esfuerzo por parte de todos los actores -sociales, económicos y políticos- para reconstruir su propio comportamiento territorial y sus espacios vividos.

Estos cambios que suceden a nivel global, presentan rasgos particulares en cada ámbito territorial. No obstante, también presentan características homogéneas que se reiteran, en mayor o menor medida, en los diversos rincones del planeta. Es ejemplo de esto, como explica Font (2007), la aparición de dinámicas de carácter urbano que se difunden en el territorio y que adquieren crecientemente características metropolitanas, en cuanto al uso y consumo del suelo, como también a las relaciones entre los diversos núcleos de urbanización. Podemos identificar así la existencia de una nueva "metropolización del territorio".

En este contexto, las ciudades portuarias metropolitanas afrontan diversas modalidades de reconversión ribereña. Los frentes fluviales se posicionan como espacios de oportunidad frente a los cambios en los modos de producción y en las estructuras industriales. Por lo cual, en la presente investigación se toma como caso de estudio la ribera metropolitana de Rosario, Argentina. ${ }^{3}$ La misma constituye un caso representativo de estudio, ya que, este frente fluvial se reposiciona a nivel internacional de un modo particular a partir de la década de 1980. Las presiones exógenas del capital, junto a las trasformaciones del Estado nacional y a nuevas condicionantes geopolíticas claves, ocasionan que el mismo se convierta en uno de los polos de exportación de oleaginosas más importantes del mundo. De manera que, esta franja fluvial se

3 Se delimita como ámbito territorial de estudio al frente fluvial del río Paraná de la Región Metropolitana de Rosario, desde la desembocadura del río Carcarañá hasta el Arroyo del Medio, en su margen occidental -provincia de Santa Fe- y la jurisdicción de la localidad de Victoria en el margen oriental -provincia de Entre Ríos-, abordando el territorio insular intermedio. 
fortalece como uno de los principales centros productivos de la economía nacional. Como plantea Pablo Ciccolella (2011), actualmente Rosario parecería ser el lugar más competitivo del país, especialmente por la dotación de infraestructura comunicacional a través del puente Rosario-Victoria y la conexión que posibilita con Brasil.

Estos nuevos modos de desterritorialización-reterritorialización se producen especialmente a partir de la década de 1980 y hasta la actualidad. Se considera que este período resulta nodo coyuntural en las transformaciones de esta región, no sólo por los cambios que posibilita el retorno de la democracia de la Argentina en 1983 -ininterrumpido hasta hoy-, sino también por el marco global de las lógicas del mercado, el capital, los flujos de comunicación y las nuevas tecnologías que inciden de un modo particular en este territorio. La hipótesis que conduce este trabajo considera que frente a las nuevas lógicas de metropolización y a las transformaciones acontecidas en el territorio durante el período de estudio se producen nuevas dinámicas territoriales que conducen a un cambio en la definición y delimitación tradicional del Área Metropolitana de Rosario. Por lo cual, en esta pesquisa se la reemplaza por la noción de Región Metropolitana de Rosario y se sostiene, además, que el río Paraná se constituye como elemento estructurador de la misma.

La mirada del río como elemento que separa ambas provincias -Santa Fe y Entre Ríos- ha sido la perspectiva prevaleciente en la historia de la región, a pesar de la existencia de diversos reclamos de unir ambas márgenes. El Paraná, de más de $55 \mathrm{Km}$ de ancho a la altura de Rosario, ha resultado para el imaginario popular un río de una orilla. Cruzar el mismo ha significado llegar a las islas -no se era consciente de la existencia del otro territorio mediterráneo-. Por lo cual, el puente Rosario-Victoria no sólo reúne aquello que está separado, sino que además hace presente la existencia del otro lado. Si comúnmente existe la frase popular que reclama que "Rosario ha crecido de espaldas al río", sin dudas también lo ha hecho lejano a su otra orilla. En esta investigación esa mirada se invierte, ya que se considera que hay una reinvención de dicho río que posibilita esa conexión. Esta perspectiva permite generar nuevas vínculos entre ambos márgenes ribereños.

Es así que la otra orilla urbana -ciudad de Victoria- se incorpora en la nueva dinámica metropolitana del Gran Rosario. Esta unión resulta cargada de posibilidades, de desarro- 
llos conjuntos, de nuevos desafíos y oportunidades. Sin embargo, aún con el puente Rosario-Victoria inaugurado desde el año 2003, las cartografías refuerzan la idea del río como límite. Se encuentra ausente la lectura integrada de ambas riberas del Paraná, dejando a un lado la riqueza de su territorio insular intermediario. Esto se evidencia también, en la escasez de documentos cartográficos -propios del análisis territorial- que registren simultáneamente ambas orillas del Paraná y su valioso Delta. El presente trabajo propone una nueva mirada sobre la Región Metropolitana de Rosario, a partir del análisis críticointerpretativo de las nuevas dinámicas territoriales que hasta ahora no han sido estudiadas de forma integrada con el enfoque propuesto en la misma.

\section{Procesos de des-reterritorialización en los ámbitos metropolitanos}

\section{Dinámicas de territorialización contemporánea}

A finales del siglo $X X$, como plantea Renato Ortiz (1998), se cristalizan un conjunto de fenómenos políticos, económicos y culturales que trascienden los pueblos y las naciones. Éstos nos permiten hablar de "globalización de las sociedades y mundialización de la cultura".
En este contexto se producen nuevos procesos de producción territorial -y aceleración de existentes- que generan nuevas lógicas de deconstrucción y reconstrucción espacial de nuestros ámbitos cotidianos. Esta articulación de deconstrucción-reconstrucción, como explica Edward Soja (2008:305), sugiere algunas claves para explicar los procesos de reestructuración territorial contemporánea. Es decir, la interacción entre desterritorialización -D- y reterritorialización -R- permite la más reveladora "comprensión de la nueva economía cultural global y la nueva política cultural de la representación y de la identidad". Según sostiene Rogerio Haesbaert (2011), la territorialización -T- integra el proceso de dominio -político/económico- y el de apropiación -simbólico/culturaldel espacio por parte de los diversos actores a través de un complejo y variado ejercicio de poderes.

Cada individuo requiere como recurso básico territorializarse. En este sentido, se considera que el uso intensivo del territorio, como simple ámbito funcional de actividades y de flujos del mercado global, tiende a desdibujar la identidad del lugar y la relación de los habitantes con el mismo. Es decir, el territorio se libera, cada vez más es utilizado como soporte de actividades y funciones económicas que son inde- 
pendientes a las relaciones del lugar y su cultura. En palabras de Carlos Reboratti (2001:148): "para muchos, la globalización ha significado la desterritoralización de muchos procesos; para otros, se trata en realidad de una re-territorialización, la adquisición de una nueva dimensión espacial, unida fuertemente al tiempo y a la velocidad de los cambios. Ya sea que aceptemos una u otra idea (o ambas), todos parecieran estar de acuerdo en que el territorio tiene un nuevo significado y un nuevo grupo de actores". De este modo, el abordaje analítico de la tríada T-D-R, nos permite re-abordar al territorio como materia configurada y significada por la cultura a partir de las posibilidades originarias que ha brindado el medio natural articulado a los procesos contemporáneos.

\section{Nuevas lógicas metropolitanas}

Dados los procesos que suceden en las últimas décadas, diversos autores (Font, 2007; Indovina, 2007; Magnaghi, 2011) plantean que, en términos generales, se produce una desestructuración de la ciudad tradicional por nuevas estructuras que responden a demandas y modelos económicos -especialmente en un marco de políticas liberales y de desregulación-. Es así que, se identifican tendencias comunes que caracterizan a los nuevos ámbitos metropolitanos. Entre ellas podemos enumerar:

1. La descentralización de las actividades industriales, que tienden a concentrarse en la periferia metropolitana;

2. Se registra un mayor incremento poblacional en las localidades distantes al centro metropolitano -que decrece en población-, dado que se convierten las segundas residencias en primeras.

3. Aumentan los componentes residenciales en territorios cada vez más distantes;

4. Existe un notable incremento de la movilidad pluridireccional -tanto de mercancías como de personas-;

5. Un mayor uso intensivo de los espacios naturales; y

6. Nuevas morfologías espaciales. Se establecen nuevas relaciones y modalidades de producción territorial $^{4}$. Es distintivo de esta nueva dinámica metropolitana tanto la transformación interna de los núcleos urbanos como también su dispersión por el territorio.

4 En relación a las producidas a mediados del siglo XX. 
Se producen nuevas configuraciones, interrelaciones e interdependencias más amplias y diferentes a las existentes anteriormente. Según explica Francesco Indovina (2007:23): "Lo que se ha dado en llamar metropolización del territorio tiene, en un cierto sentido, el poder de reproducir la ciudad (...), es decir, preservar en una situación nueva un contexto de intercambios no solo económicos, el lugar donde se crean y recrean continuamente los "mestizajes" culturales, se multiplican las relaciones sociales, se manifiestan grandes contradicciones y se innova la vida económica, social y cultural. En suma, se salva y se renueva el "nicho ecológico" de la especie humana. La estructura territorial metropolitana, además, se puede asumir como expresión de la cultura contemporánea (...) el proceso en curso, no solo urbano sino también social, se caracteriza por la tensión en la agregación; el sujeto se reagrega, de la misma forma que se recompone el fragmento; la mezcla de las diferencias, en los diferentes niveles y en los diversos contextos, aparece como una fuerte aspiración de la contemporaneidad, es evidente que por su carga innovadora precisamente, produce pulsiones de rechazo".

Franceso Indovina, asimismo, sostiene que si por área metropolitana se entiende a un territorio cuyas partes están integradas entre sí vinculadas según funciones diversas y presenta una estructura jerárquica, al proceso que se disgrega del mismo se puede llamar metropolización del territorio. En las últimas décadas, este fenómeno adquiere nuevas modalidades a partir de las transformaciones del sistema económico y social, como también de las nuevas relaciones establecidas en dichas áreas. ${ }^{5}$ El crecimiento acelerado de los ámbitos metropolitanos, como explica Ciccolella (2011), resulta una de las tendencias más fuertes, en todo el mundo, especialmente a partir de los 90s. En este período, estos espacios retoman el crecimiento en relación a la concentración del poder económico, de las actividades productivas estratégicas y del producto bruto geográfico, siendo central el rol del capital en la transformación de los mismos.

5 Francesco Indovina agrega que "Las áreas metropolitanas tradicionales tienden a pasar de una jerarquía hard a una jerarquía soft (Indovina, 1999). De hecho, los vínculos del centro con el resto del territorio tienden a modificarse; los movimientos de personas no son solo monodireccionales (de la periferia al centro), sino que se convierten en pluridireccionales, también las del centro a la periferia, como resultado de la difusión en el territorio no solo de población sino también de actividades, funciones comerciales, servicios, etc. La tendencia, pues, ya no es concentrar en un único punto (ciudad central) las funciones principales, las económicas y los servicios superiores, sino más bien distribuir en el territorio amplio puntos de especialización, diversificados pero, precisamente, integrados entre sí, que hacen, por decirlo así, un todo" (Indovina, 2007). 
Es así que, frente a la aceleración de los procesos existentes y el desarrollo de nuevas dinámicas metropolitanas, surge la necesidad de afrontar de un modo distinto la producción territorial contemporánea. Las definiciones tradicionales de zona metropolitana o área metropolitana, que delimitan el territorio principalmente desde condicionantes físicas -en cuanto a contigüidad y continuidad de los núcleos urbanos, por ejemplo- resultan insuficientes en la actualidad dadas las nuevas lógicas metropolitanas. Como plantea el Plan Estratégico Metropolitano de Rosario (2004): "el territorio metropolitano no es algo dado a priori, sino un proceso de construcción social, resultado de las estrategias de organización de los actores y del tipo de instituciones que conforman el territorio. Esto implica mirar, considerar al territorio como una estructura compleja e interactiva, en el cual, el contenido define el continente (límites, dimensiones y otros atributos geográficos) (...) el territorio se constituye en el espacio privilegiado de la interacción y del conflicto, donde se desenvuelven múltiples relaciones sociales que lo identifican como tal. En este concepto puede enmarcarse el análisis del espacio metropolitano".

De manera que, el concepto de Área Metropolitana dependiente del binomio centro-periferia y caracterizado por la ciudad central -que en su proceso de expansión tiende a absorber otros poblados-, como plantea Borja (2012) resulta desbordado por el desarrollo de una urbanización multiescalar. Por lo cual, como sostiene Castells (2013), es más propicio el término de Región Metropolitana para referirnos a estas nuevas interconexiones territoriales. Este autor explica que la nueva modalidad de Región Metropolitana ${ }^{6}$ surge a partir de dos procesos inter-vinculados. Por un lado, la descentralización de las grandes ciudades, que se expande a las zonas adyacentes; mientras que, por el otro lado, se sucede la interconexión de pueblos preexistentes cuyos territorios se integran a través de las nuevas capacidades de comunicación. De manera que, Manuel Castells (2013), citando a Pain y Hall (2006:3), caracteriza a estas regiones como: "una forma nueva [que incluye] entre 10 y 15 ciudades y pueblos, físicamente separados pero funcionalmente interconectados, agrupados alrededor de una o más ciudades grandes, espacialmente distantes, que atraen enorme poder económico de una nueva división funcional de la fuerza de trabajo. Dichos lugares existen como entidades separadas en las cuales la mayoría de

6 Manuel Castells define este concepto en relación al acuñado por Kathy Pain y Peter Hall (2006) en "The Polycentric Metropolis". 
los residentes trabajan en la localidad y la mayoría de quienes trabajan son residentes de ese lugar (...) y como región funcional que se encuentra conectada por redes de transporte y comunicación por las que fluyen personas, bienes, servicios e información".

Por lo cual, en la presente investigación utilizamos el concepto de Región Metropolitana, a fin de abordar los múltiples procesos dinámicos y complejos que suceden en el territorio interconectado. Analizar dichos procesos, requiere un abordaje interescalar que permita la articulación entre los distintos niveles de interacción; es decir, que posibilite una comprensión integral a partir de una exploración de las lógicas propias de lo local. La mirada metropolitana se constituye como una perspectiva de oportunidad para comprender e interpretar las problemáticas y potencialidades presentes en nuestros territorios contemporáneos. Si bien por un lado los rasgos de los ámbitos metropolitanos presentan analogías a escala global, por el otro, poseen especificidades propias. Los procesos de globalización, las nuevas condiciones de mercado, los procesos productivos, las nuevas tecnologías, entre otros factores, plantean a nivel global nuevas formas de organización y relaciones territoriales. Sin embargo, los condicionantes locales asumen y transforman a su vez nuevos vínculos y particularidades en cada territorio metropolitano. De este modo, las nuevas dinámicas globales, junto a las peculiaridades locales, producen características propias en el mencionado proceso de metropolización.

\section{De Área Metropolitana a Región Metropolitana de Rosario: Nuevas perspectivas territoriales}

\section{Antecedentes}

El ámbito territorial estudiado en la presente investigación se encuentra en la pampa ondulada de la República Argentina. El mismo se caracteriza por la conjunción de tierra y agua, presentando extensas llanuras interrumpidas verticalmente por el caudaloso río Paraná y seccionadas transversalmente por diversos meandros y cursos de agua. Las pendientes suaves, la fertilidad de la tierra junto al clima templado-húmedo favorecen las actividades agrícolas ganaderas -resultando uno de los espacios productivos rurales más importantes del país-. El frente ribereño occidental del Paraná presenta características geológicas y topográficas excepcionales que favorecen las actividades portuarias e industriales. Las barrancas de gran altura y el importante calado del río confieren características de puerto natural (Figura 1). 





La conformación de Rosario y su región se encuentra favorecida por la privilegiada posición geográfica y estratégica junto a diversos condicionantes geopolíticos claves. Resulta así un punto coyuntural a orillas del río Paraná. Si bien originalmente este curso fluvial solo era utilizado para provisión de agua y pesca, posteriormente se constituye en el factor principal de transformación regional a partir del impulso portuario. La pampa y el río resultan así, desde época temprana, los componentes esenciales de la riqueza de este territorio. Este espacio fluvial se encuentra sujeto, desde sus primeras marcas antrópicas, a numerosas apropiaciones y diversas intensiones "tanto míticas como políticas". Como plantea Corboz (1983:14-35), "el territorio no es un dato, sino el resultado de diversos procesos". Es decir, el mismo "es objeto de una construcción (...) en consecuencia, el territorio es un proyecto". Su transformación a través del tiempo involucra diversos actores, intereses, planes e intenciones. Varias tensiones entran en juego en la definición de sus límites y fronteras -vinculados al poder y apropiación del mismo-: la conquista del territorio, las delimitaciones provinciales, la lucha por la definición del Estado nacional y la capacidad de control del espacio concreto.
Son numerosos los proyectos territoriales que, a modo de capas, se superponen en este palimpsesto y contribuyen a la construcción sucesiva del mismo, por ejemplo: el proyecto de la Confederación Argentina, que conforma una red de nodos claves en el país de "tierra adentro"; el sistema ferroviario, estructurador central del territorio en el siglo XIX, y su articulación con la colonización agrícola y con el sistema portuario; la trama de la red vial; entre otros. Asimismo, en relación a estos múltiples proyectos sucesivos, con el transcurrir de las décadas se identifica y fortalece la necesidad de una mirada ampliada de este espacio geográfico -que supere las diversas jurisdicciones existentes-. Es así que, desde los primeros pasos de la planificación local, la perspectiva territorial se hace presente.

La toponimia para referirse a este territorio es variada a lo largo de su historia: originalmente denominado "Pago de los Arroyos", posteriormente se identifica como: Rosario y su zona de extensión, Rosario y su zona de influencia, Región de Rosario, Gran Rosario, Área Metropolitana, Región Metropolitana de Rosario, entre otras. No obstante, a pesar de las diversas miradas que proponen nuevos términos y delimitaciones, 
ROSARIO Y SU ZONA DE EXTENSIÓN - 1935



REGIÓN METROPOLITANA DE ROSARIO - 1991

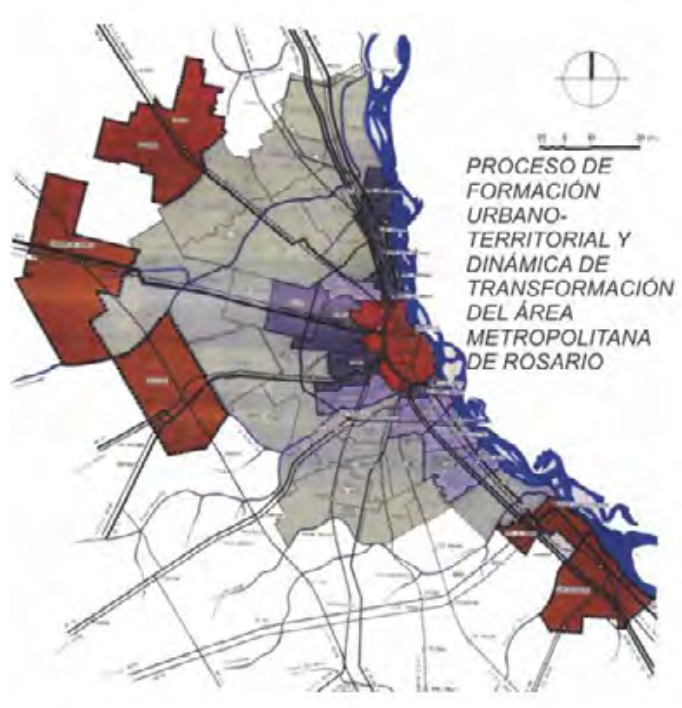

ÁREA METROPOLITANA DE ROSARIO - 1971

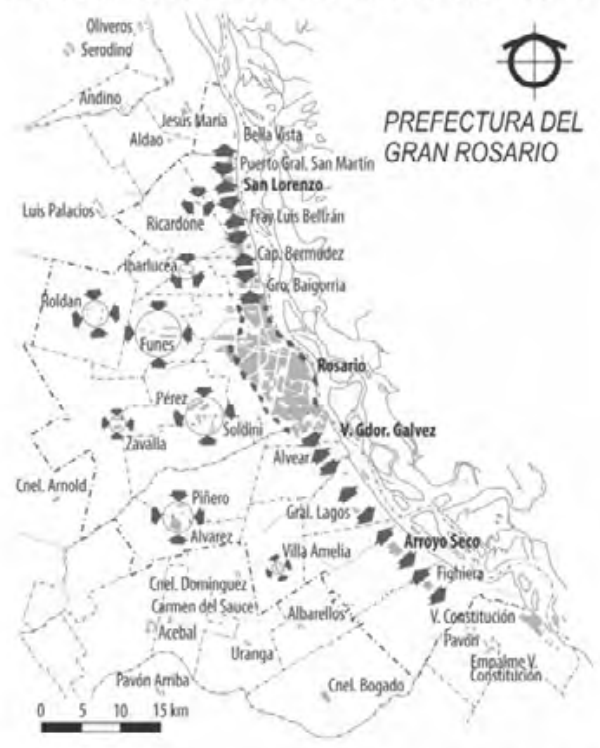

ÁREA METROPOLITANA DE ROSARIO - 2014

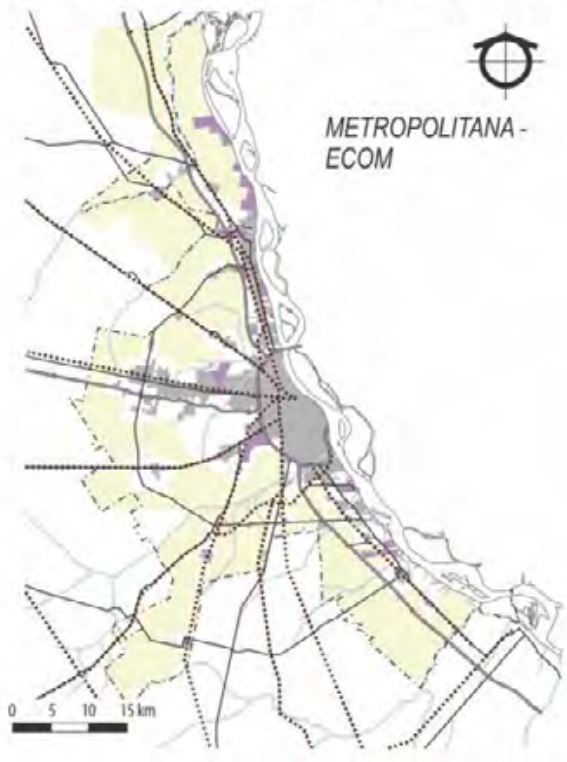


aquella que ha prevalecido con más fuerza hasta la actualidad, en materia de planificación territorial, es la de Área Metropolitana de Rosario $(A M R)^{7}$-desde su definición por la Prefectura del Gran Rosario en 1969 hasta la actualidad a través del ECOM - Ente de Coordinación Metropolitana(Figura 2).

Si bien la delimitación de las jurisdicciones que forman parte del AMR es variable según los distintos estudios y proyectos, todas coinciden en afirmar al río Paraná como límite este de la misma. Sin embargo, frente a los diversos procesos de territorialización que suceden desde las últimas décadas, consideramos en la presente investigación que dicha mirada estática resulta restrictiva. A continuación nos detenemos a analizar los cambios en el ámbito territorial de estudio que requieren ampliar la mirada en el estudio de este territorio.

\footnotetext{
7 Es importante resaltar que en la historia de la planificación territorial de Rosario, para la delimitación de su área metropolitana no se han tomado los mismos criterios utilizados en otras provincias Argentinas -como Buenos Aires, por ejemplo-. Factores como la conurbación y la contigüidad física sólo se verifican desde Puerto General San Martín a Villa Gobernador Gálvez -en el frente costero-, mientras que otras localidades ribereñas como Alvear, Pueblo Esther, General lagos, Arroyo Seco -entre otras-, dadas sus interacciones y vínculos con el resto de este territorio, han sido consideradas históricamente partes del área a pesar de no conformar un continuo urbano.
}

\section{Territorializaciones metropolitanas desde las últimas décadas del siglo XX}

Como planteamos en el primer apartado, frente a los cambios globales de las dinámicas económicas y del capital se producen nuevas desreterritorializaciones en cada ámbito particular. Esto se refleja, especialmente desde las últimas décadas, en el frente fluvial metropolitano de Rosario. Si bien, desde mediados del siglo XX esta ribera constituye un polo portuario-industrial exportador de gran consolidación, ésta se readapta y consolida su perfil estratégico ya sea a partir de los nuevos desafíos del mercado internacional como del contexto político-económico local.

Es así que se identifican diversos cambios en los modos de producción. Por ejemplo, el sistema agrícolaganadero atraviesa en el período de estudio una profunda transformación frente a la extensión del cultivo de soja -junto a la incorporación de la soja transgénica resistente al herbicida glifosato- y la siembra directa, incrementando ampliamente la exportación de la misma y sus derivados. Como explica Pierri (2006), es el nuevo mercado mundial el que en la década de 1980 posibilita este "boom de la soja". De manera que, del millón de toneladas exportadas 
en la década de 1970, llega en los últimos años a superar los treinta millones de toneladas, convirtiendo así a la Argentina en el principal exportador mundial de oleaginosas y subproductos.

Asimismo, como registra la Bolsa de Comercio de Rosario, aproximadamente el $78 \%$ de las exportaciones nacionales de granos, aceites y subproductos se despachan por las terminales portuarias localizadas en la ribera metropolitana aquí estudiada. La misma, según explica Julio Calzada (2012), resulta el complejo oleaginoso más importante a nivel mundial, por las características que presentan sus instalaciones y por exportar 134.000 ton/día. De manera que, en las últimas décadas, se instalan en este frente costero nuevas plantas de crushing con grandes capacidades cada una, como también otros complejos productivos industriales y/o portuarios. Es de remarcar, que la mayoría de los mismos son de capitales internacionales; son ejemplo de esto las terminales de: Louis Dreyfus Commodities, Cargill, General Motors, Petrobras, Alfred Toepfer S. A., Akzo Nobel, entre otras.

En este contexto, de gran crecimiento y relación de los mercados en el marco de una creciente globa-

lización, comienzan a establecerse diversas estrategias y propuestas articuladas entre países vecinos para potenciar las capacidades de cada mercado particular. De este modo, en 1991 se crea el Mercado Común del Sur-MERCOSUR- entre los países de Argentina, Brasil, Paraguay, Uruguay y Venezuela, con el fin de complementar sus economías y mejorar la inserción internacional de América del Sur en el mercado global. Asimismo, desde los últimos años de la década de 1980 y los primeros de 1990, se establece el proyecto de la Hidrovía Paraná-Paraguay. El mismo constituye un sistema navegable de gran envergadura a través del cual conecta el Puerto de Cáceres en Brasil, con los diversos nodos portuarios de los ríos Paraguay, Paraná y de la Plata, hasta el Puerto de Nueva Palmira en el Uruguay. Se integran así los países de Argentina, Bolivia, Brasil, Paraguay y Uruguay. El sector ribereño aquí estudiado resulta de gran importancia para la hidrovía, especialmente porque es en este sector -desde la localidad de Puerto General San Martín- que se alcanzan los 34 pies de calado.

De manera que, frente a este gran crecimiento regional, el sector de estudio se readapta para dar respuesta a dichas potencialidades; 
por ejemplo, en materia de infraestructura. Si bien por un lado existen demoras y retrasos en proyectos necesarios ya planificados -como la reestructuración ferroviaria y la ejecución de nuevos nodos de articulación vial entre las diversas arterias-, por otro lado se realizan infraestructuras claves para contribuir a la competitividad regional, como es el caso de la construcción del Puente Rosario -Victoria. Esta conexión directa entre ambas orillas del Paraná, a la altura de la ciudad de Rosario, es anhelada durante siglos -podemos fecharlo desde mediados del siglo XIXNo obstante, luego de numerosos proyectos, propuestas y reclamos a través de los años, es recién en 1998 que comienza la obra, inaugurándose en el año 2003. La misma representa un eslabón clave del sistema de transporte multimodal.

A partir de la inauguración de este puente se generan nuevas y diversas reterritorializaciones. En palabras de Heiddeger (1951), "el puente, con las orillas, le aporta a la corriente las dos extensiones de paisaje que se encuentran detrás de estas orillas". El puente reúne, coliga, hace aparecer ambas márgenes. Es justamente por el puente que una deja de ser indiferente a la otra, y posibilita una nueva trama de potencialidades, de nuevas relaciones impensadas antes de su concreción física. De manera que, en la década del 2000, la orilla oriental del Paraná se inserta en la dinámica metropolitana de Rosario. La localidad entrerriana de Victoria, único centro urbano en la costa oriental de este sector, adquiere una nueva dialéctica con el frente fluvial occidental. Por lo cual, esta conexión posibilita concretar físicamente nuevos vínculos, relaciones y potencialidades en las distintas escalas territoriales, tanto desde el ámbito local -la transformación que sucede en la propia ciudad de Victoria, por ejemplo,-, a nivel regional -a partir de las nuevas dinámicas interprovinciales que se generan entre ambas orillas del Paraná- y a escala global, dados los nuevos lazos e interrelaciones entre los mercados de Chile, Brasil y Uruguay, como su conexión fluvial al resto del mundo -es de destacar el Corredor Biocéanico Atlántico - Pacífico-. De este modo, consideramos que frente a los diversos procesos de transformación que toman lugar en esta ribera metropolitana en las últimas tres décadas, es necesario ampliar la mirada de las delimitaciones históricas del AMR. Por lo cual, también se plantea dejar de lado la noción del río Paraná como límite, a fin de identificarlo en cambio como enlace territorial. 


\section{Potencialidades de una mirada ampliada: un río de dos orillas}

"Y únicamente al cabo de un momento, el observador se da cuenta de que, al alzar la vista hacia el horizonte, en la vaciedad singular de la extensión que se despliega ante sus ojos, falta también aquello que, en la configuración de todos los ríos, descansa la mirada y tranquiliza, completando la idea, el arquetipo de la noción misma de "río": la orilla opuesta" (Saer, 2010: 29).

Frente a los cambios desarrollados, a partir de las décadas de 1980-1990, desde algunos ámbitos se propone sustituir el concepto de "Área Metropolitana" por el de "Región Metropolitana". Este cambio es propuesto originalmente por el proyecto de investigación denominado "Proceso de formación urbano-territorial y dinámica de transformación del Área Metropolitana de Rosario" 8 de 1991, dirigido por el Arq. Adrián Caballero. En el mismo se plantea que es necesario conformar una "real conciencia de la dimensión metropolitana", para lo cual se requiere plantear una reformulación

8 El mismo surge de un convenio entre la Sub-secretaría de Planeamiento y Control de Gestión de la Provincia de Santa Fe, la Facultad de Arquitectura, Planeamiento del perímetro metropolitano determinado. De este modo, se cuestiona la noción misma de "Área Metropolitana" y se la reemplaza por el concepto de "Región Metropolitana". Como explica Caballero (1991:11), se propone la utilización de este término "como intento de concretar territorialmente la intención expuesta de ampliar y complejizar la noción tradicional de "área metropolitana", con la que históricamente se ha manejado, en el caso Rosario, el fenómeno expansivo de la "ciudad central" (fundamentalmente en el antecedente técnico-institucional más relevante: la Prefectura del Gran Rosario)".

De manera que se propone una nueva perimetración delimitada según los niveles reconocibles de máxima interacción. Es decir, el concepto de "Región Metropolitana" resulta "una suerte de sumatoria" de las distintas expresiones urbano-territoriales presentes en este espacio concreto (Caballero, 1991). Desde esta noción de interrelación compleja entre diversas localidades -a través de procesos económicos, sociales, culturales, políticos, etc.-, se retoma posteriormente este concepto en otros estudios y trabajos $^{10}$. No obstante, es

9 Entendida como extensión unitaria y homogénea

10 Por ejemplo en la Agencia de Desarrollo Región Rosario -ADERR-. 


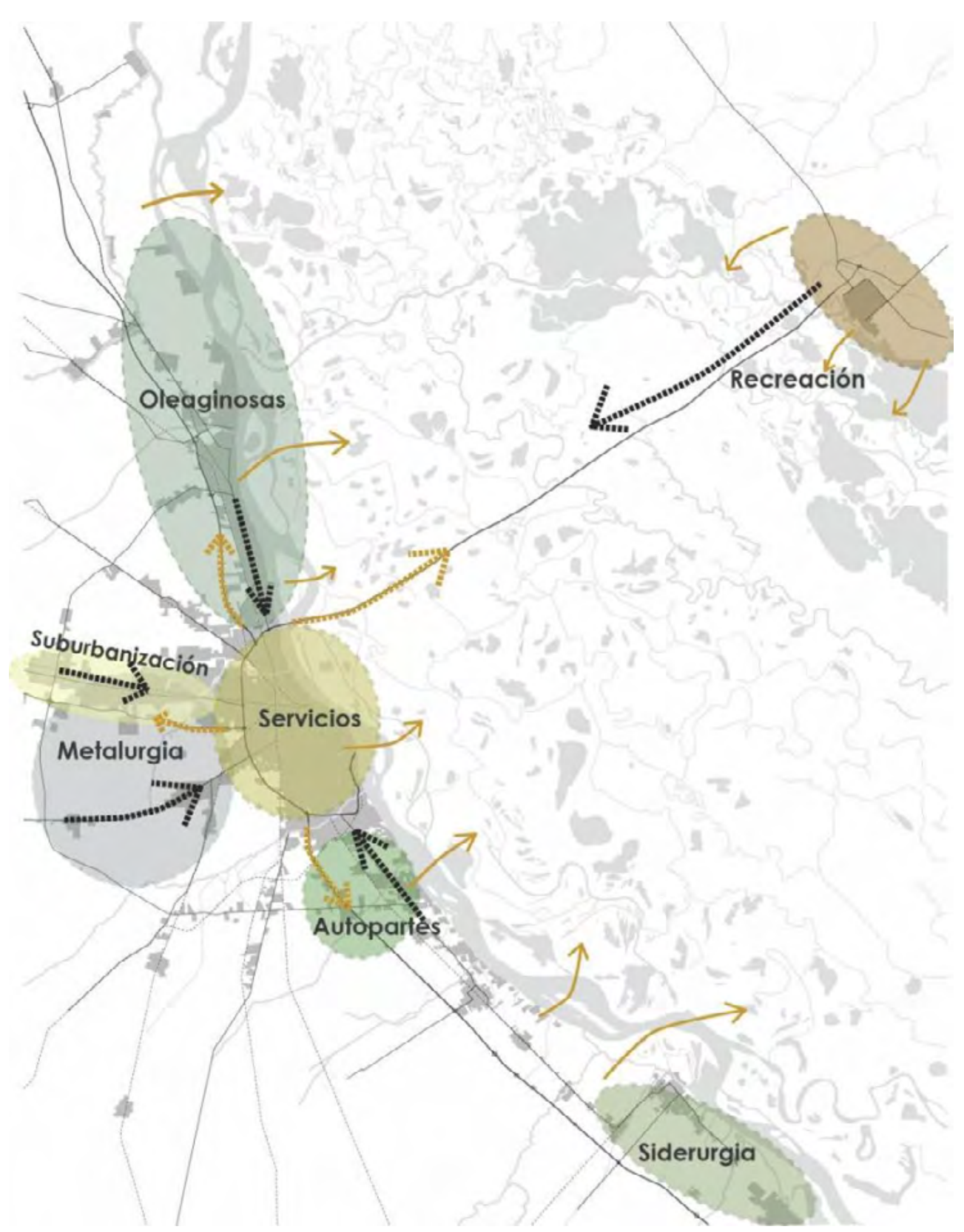

Figura 3. Nuevas centralidades y vínculos en la Región Metropolitana de Rosario. Fuente: Elaboración propia en base al Plan Estratégico Metropolitano (2004). 
de gran relevancia su utilización en el Plan Estratégico Metropolitano -PEM-, en los primeros años de la década del 2000. Este plan define a la Región Metropolitana de Rosario como "un ámbito urbano-rural que se estructura en torno a la ciudad central y se articula con otros centros urbanos que cuentan con mercados de trabajo y ámbitos comerciales propios, vinculados al centro. Incluye municipalidades relativamente alejadas de la de Rosario. Esta demarcación no es definitiva, su flexibilidad y movilidad está sujeta a las dinámicas económicas, productivas y sociales que involucran a los diferentes actores, en el proceso de construcción de un territorio metropolitano" (Municipalidad de Rosario, 2004: 17).

El PEM nuevamente considera a la dimensión metropolitana en su mayor alcance territorial, focalizándose en las diversas realidades y procesos presentes en el territorio: "la forma metropolitana presupone un espacio de unidad de la diversidad, de conjunción de varias unidades territoriales, homogéneas hacia su interior, que conforman un espacio más amplio, precisamente, signado por su heterogeneidad" (Municipalidad de Rosario, 2004:19). Se identifican así, diferentes lógicas de transformación y nuevas
7. La ciudad de Rosario como nodo central, dada la convergencia de fuerzas y flujos hacia ella; produciendo un efecto centralizador.

8. El eje articulador del río Paraná, principalmente en relación a las actividades portuarias. La ciudadpuerto por excelencia que era Rosario originalmente, se expande predominantemente hacia el norte, en primer lugar, y hacia el sur, en segundo lugar; produciendo nuevas centralidades vinculadas al flujo de oleaginosas, químicos y siderurgia -entre otros-.

9. Esto decanta en la identificación de nuevas centralidades productivas. Por lo cual, el escenario productivo regional tiene múltiples centralidades y nuevas dinámicas y relaciones.

10. Desde la perspectiva de nuevas articulaciones y proyección territorial, además del eje norte sur -que mencionábamos en el punto 2, como eje del Paraná-; asimismo, se consolida fuertemente el eje este-oeste, con la inauguración del Puente Rosario-Victoria, relacionando a la región a través del corredor bioceánico, conectando el Pacífico y Chile -hacia el oestecon la República Oriental del Uruguay, al este. 


\begin{tabular}{|c|c|}
\hline Área metropolitana de Rosario & Región metropolitana de Rosario \\
\hline $\begin{array}{l}\text { Comprende los municipios del espacio metropolitano, que } \\
\text { configuran un territorio integrado, donde se manifiesta una } \\
\text { interacción física, económica, socio-cultural y deman- } \\
\text { das político administrativas comunes. Se distinguen dos } \\
\text { escalas de definición dentro del área metropolitana: } \\
\text { La "conurbación": donde se verifican como condiciones la } \\
\text { contigüidad física de núcleos urbanos. } \\
\text { La "extensión": donde quedan incorporadas grandes } \\
\text { extensiones rurales, no existiendo contigüidad física entre } \\
\text { los núcleos urbanos pero sí una importante interacción eco- } \\
\text { nómica, social y funcional. }\end{array}$ & $\begin{array}{l}\text { Refiere a la unidad territorial inter-pro- } \\
\text { vincial que componen los municipios } \\
\text { y comunas de la totalidad del espacio } \\
\text { metropolitano, integrando municipios } \\
\text { de otras jurisdicciones provinciales que } \\
\text { mantienen fuertes vinculos económicos, } \\
\text { sociales y funcionales entre sí. }\end{array}$ \\
\hline
\end{tabular}

Cuadro 1. Definición de Área Metropolitana de Rosario y de Región Metropolitana de Rosario según anteproyecto de Ley de Creación de Regiones Metropolitanas.

Elaboración propia en base a anteproyecto de "Ley de Creación de Regiones Metropolitanas" Provincia de Santa Fe - 2007.

Dadas estas nuevas dinámicas metropolitanas, el PEM ya realiza una proyección de incorporar a Victoria -Provincia de Entre Ríos- a la Región Metropolitana de Rosario. De este modo, se identifican fuertes vínculos y relaciones de interdependencia social, productiva, económica, cultural -entre otras-, en el territorio, que exceden las jurisdicciones provinciales. A su vez, en el año 2007, la Oficina de Asuntos Metropolitanos de la Provincia de Santa Fe propone el Anteproyecto de Ley de Creación de Regiones Metropolitanas, identificando en el espacio metropolitano ${ }^{11}$ tres ámbitos de actua-

11 Definido como la unidad geográfica integrada por territorios rurales y urbanos entre los que existen vínculos físicos, funcionales, económicos y sociales. ción ${ }^{12}:$ 1. Conjunto de Municipios ${ }^{13} ; 2$. Área Metropolitana y 3. Región Metropolitana. (Cuadro 1)

Como vemos, este proyecto de ley no suplanta el concepto de "área" por el de "región", sino este último amplía la mirada del espacio metropolitano, a fin de integrar la totalidad del mismo -más allá de los límites de las jurisdicciones provinciales- a partir de los fuertes

12 Se consideran "ámbitos de actuación metropolitana" aquellos que se constituyen para desarrollar acciones de cooperación y coordinación, planificación y ejecución de políticas, programas y proyectos a través de figuras u órganos de gestión inter-municipal o supra-municipal.

13 "Se refiere a dos o más municipios que pertenecen al espacio metropolitano, vinculados por intereses comunes, sin que sea condición necesaria la cercanía o la contigüidad espacial" (Oficina de Asuntos Metropolitanos, 2007). 
vínculos económicos, sociales y funcionales existentes. En este sentido, esta investigación retoma esta definición de Región Metropolitana a fin de abordar una mirada ampliada el territorio, que no se limite a las jurisdicciones políticas sino que incluya a los diversos vínculos económicos, sociales y funcionales.

Es así que, en el período de estudio, las relaciones inter-jurisdiccionales se aceleran y consolidan, creando nuevas oportunidades y potencialidades a la región. Ambas orillas del río comienzan a entablar una dialéctica fluida tanto entre sus márgenes como con el territorio insular. La ciudad de Victoria ${ }^{14}$

14 La ciudad de Victoria resulta cabecera del departamento homónimo, el cual posee una superficie de $6.822 \mathrm{Km}^{2}$. El mismo presenta un grado muy bajo de urbanización, dado que es Victoria el único municipio de dicho departamento. El resto de los poblados son centros rurales de población. Esta jurisdicción presenta un alto porcentaje de suelo rústico, del cual mayoritariamente corresponde a territorio insular: más de $3.760 \mathrm{Km}^{2}$ de islas han sido incorporadas a la jurisdicción de Victoria mediante la Ley Provincial de Entre Ríos N 8.855/94. Esta ciudad presenta una condición particular de su emplazamiento en relación al resto de las localidades urbanas de la Provincia de Entre Ríos. Las ciudades entrerrianas más cercanas a Victoria son: hacia el norte, Diamante a $80 \mathrm{Km}$; hacia el sur, la localidad de Gualaguay a 103 Km; y hacia el este, Nogoyá a 44 Km de distancia aproximadamente. Es de remarcar además, que estas tres localidades presentan una escala y densidad poblacional relativamente análoga a Victoria. La ciudad de Paraná, capital de la provincia de Entre Ríos, es la localidad de mayor jerarquía y de mayor cantidad de población entrerriana -con 247.863 habitantes-, y se encuentra a 118 $\mathrm{km}$ de Victoria. Sin embargo, Victoria se sitúa a $60 \mathrm{Km}$ de la ciudad de Rosario a partir de la inauguración del Puente Rosario-Victoria en el año 2003, hecho que desencadena una profunda transformación regional. -único componente urbano de la orilla oriental frente a Rosario-, se incorpora a la dinámica de la Región Metropolitana de Rosario especialmente desde la construcción del puente RosarioVictoria. Por ejemplo, desde fines de la década de 1990, dicha localidad entrerriana atraviesa diversas transformaciones a partir de su reposicionamiento en este territorio. Por lo cual, se producen nuevos emprendimientos vinculados principalmente al turismo y a la recreación, como también vinculados a ofrecer nuevas áreas residenciales asociadas al paisaje del campo, del río y sus actividades náuticas. El mercado inmobiliario se ve prontamente alterado frente a la alta expectativa de la nueva conexión interregional. Asimismo, los cambios en los flujos de movilidad de personas no sólo involucran a los victorienses que se trasladan frecuentemente a Rosario para acceder a los servicios ${ }^{15}$ ofrecidos por la gran ciudad, sino también se produce el efecto inverso. Es decir, rosarinos y habitantes de la orilla occidental acuden a Victoria periódicamente, ya sea en búsqueda de nuevos modos de habitar -asociados al paisaje ribereño e insular- o atraídos por nuevas ofertas turísticas-recreativas (Figura 4).

15 Principalmente educación -universitaria-, salud y servicios terciarios. 

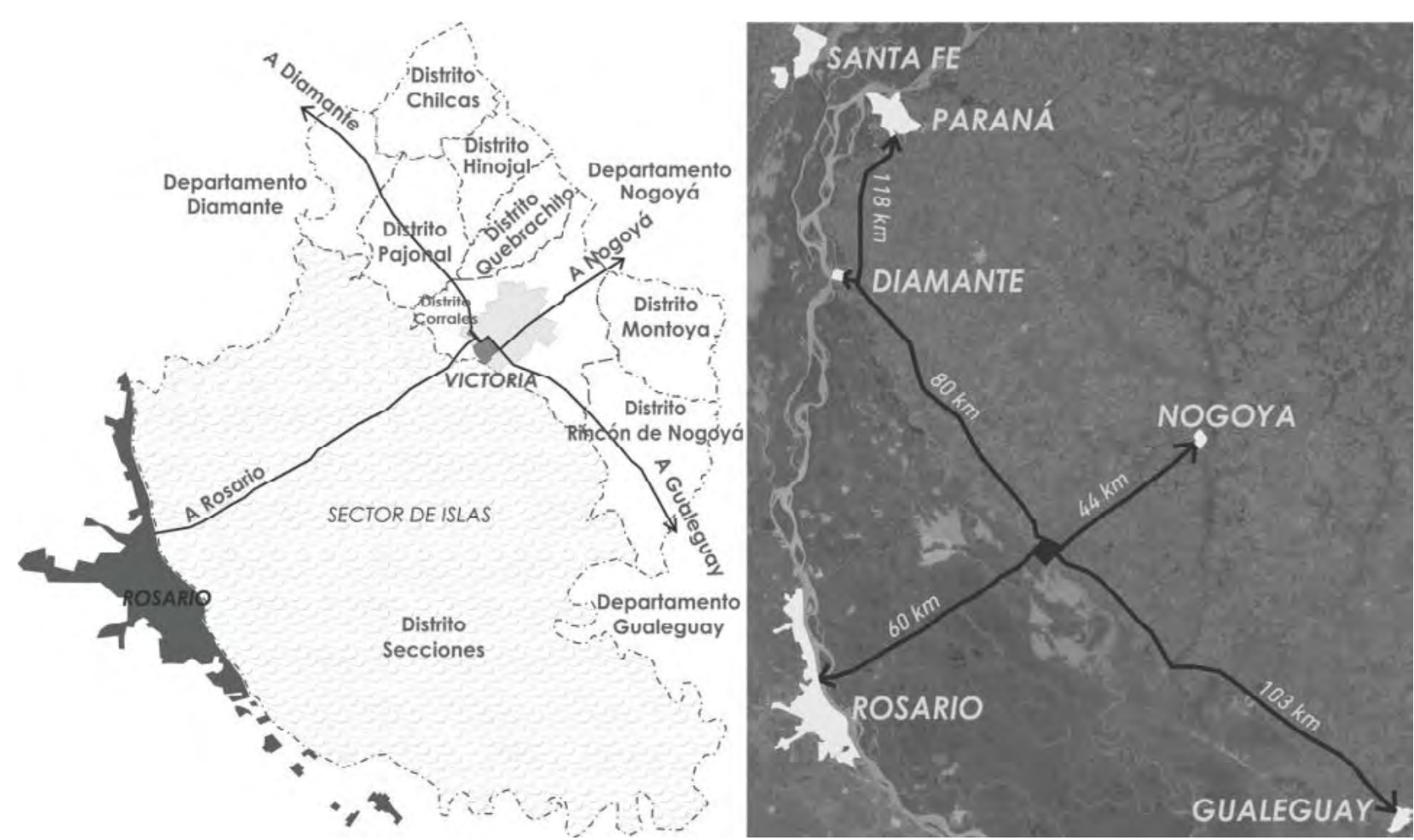

Figura 4. Jurisdicción del Departamento Victoria (Izquierda)-

Entre Ríos. (Derecha): Distancias de la ciudad de Victoria en relación a las localidades más cercanas.

Fuente: Elaboración propia.

Victoria reposiciona su rol en el territorio, convirtiéndose en un nuevo centro turístico para la región, especialmente a partir de su atractivo ribereño e insular. En este sentido, la costanera es el principal espacio elegido para comenzar sus mayores transformaciones, inaugurándose en el 2005 el balneario principal de la ciudad -estando aún en curso el proyecto del Plan Maestro del Frente Costero-. Asimismo, los emprendimientos asociados al turismo han sido punta de lanza de este proceso: en el 2004 se inaugura el Hotel 5 estrellas Sol Victoria Hotel-Spa-Casino ${ }^{16}$, siendo el primer hotel de esa jerarquía en toda la región ${ }^{17}$; como el Casino de Victoria, inaugurado en el 2005 -resulta también el primer casino del Gran Rosario y

16 A su vez, se incrementa en gran medida la plaza hotelera.

17 La ciudad de Rosario inaugura su primer hotel 5 estrellas "Ros Tower" en el año 2006, y el hotel "City Center" en el año 2009. 


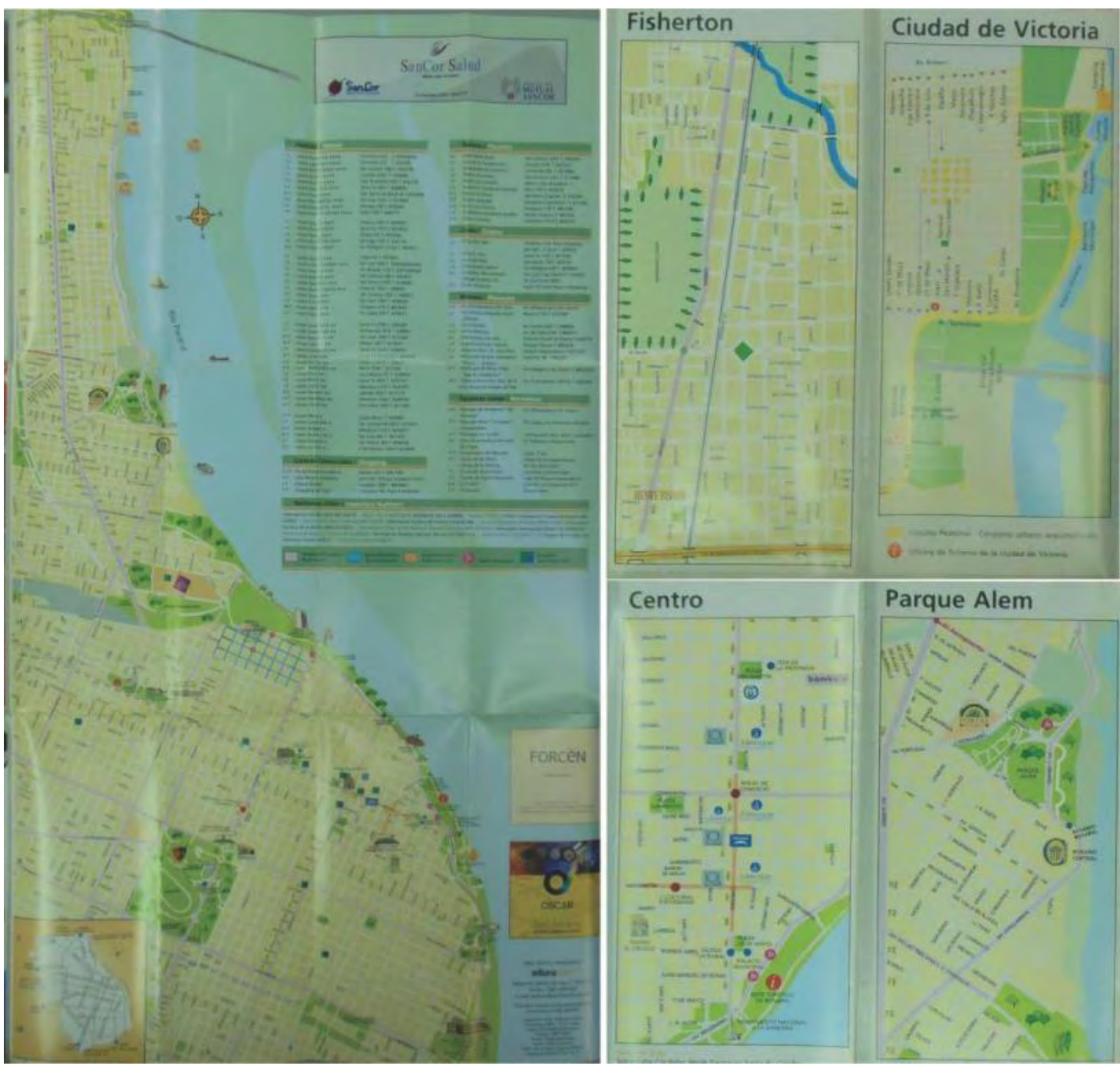

Figura 5. Mapa turístico de la ciudad de Rosario - Ente Turístico de Rosario MR - Año 2005 (aprox.).

Fuente: Mapoteca de la Biblioteca Nacional de la República Argentina

alrededores-. También así, el parque temático y termal Victoria del Agua -de 200 Has aproximadamente- se constituye en un gran foco de atracción turística. Resulta ejemplo de esto, los planos turísticos de Rosario que incorporan como paseos recreativos a la ciudad a Victoria. 
En la figura 5, vemos el "Mapa de Rosario" desarrollado por el Ente Turístico en la primera década del 2000, en el cual se identifican dos particularidades que contribuyen a la argumentación de la relevancia de la ribera y de la integración de las dos orillas. Por un lado, este plano solo presenta el frente ribereño de la ciudad -desde la cabecera del mencionado puente hasta la Ciudad Universitaria-. Por el otro lado, expone en una mayor escala los focos turísticos principales de Rosario, cartografiando cuatro paseos recomendados: el centro de la ciudad; el Parque Alem; Fisherton y la ciudad de Victoria. Es decir, incorpora a esta última como un paseo más de Rosario.

Asimismo, si bien el gran territorio insular ${ }^{18}$ frente a la ciudad de Rosario corresponde a la jurisdicción de Victoria, la interconexión entre la ribera occidental del Paraná y las islas frentistas a esta orilla presenta un alto nivel de conectividad. Fundamentalmente en las últimas décadas, la vinculación de los habitantes

18 A excepción de algunas islas que corresponden a la jurisdicción de Santa Fe -cuya superficie es mínima en relación a las correspondientes a Entre Ríos-. rosarinos $^{19}$ con el delta del Paraná, como de los habitantes isleños con Rosario, registra una relación tan estrecha y dinámica que para el imaginario colectivo las islas forman parte de Rosario. Éstas son frecuentadas asiduamente por los habitantes del territorio occidental al río. La flota náutica se incrementa vertiginosamente, como también las actividades y emprendimientos insulares. Además, las cinco bajadas del puente a las zonas de islas posibilitan una apropiación mayor de este territorio isleño, pero no solo desde la perspectiva recreativa y de disfrute, sino también en gran medida desde la producción -ganadería, apicultura, entre otras-. Esta conexión este-oeste se constituye como un eje clave de intercambio de flujos inter-regionales -ya sea de movilidad de mercancías y de personas-. De este modo, se registran diversas transformaciones en las lógicas del Área Metropolitana de Rosario, produciéndose nuevas relaciones interprovinciales que posibilitan nuevas dinámicas locales, regionales e internacionales. Ampliar la escala de la mirada posibilita un cambio en el abordaje y desarrollo de las potencialidades presentes en los territorios contemporáneos.

19 Como del resto de las localidades de la orilla occidental del Paraná de la Región Metropolitana de Rosario. 


\section{Conclusiones}

En la presente investigación consideramos que los límites jurisdiccionales abstractos condicionan la mirada sobre el territorio. Es decir, las divisiones políticas parecen materializarse y constituirse en barreras físicas que impiden la visión global de los procesos intervinientes en el espacio concreto; los cuales comprenden otras lógicas y exceden dichos límites. Justamente, muchas veces la visión taxativa sobre el territorio impide dar respuestas eficaces a conflictos comunes que superan las jurisdicciones intervinientes -por ejemplo, como sucede con la problemática ambiental vinculada al Delta del Paraná-. En este sentido, retomando a André Corboz (1983:36), afirmamos que el territorio se encuentra en "perpetua metamorfosis", cambia sus relaciones estableciendo nuevos vínculos: "el territorio se extiende más allá, siempre diferente a lo que sabemos de él, a lo que vemos de él, a lo que queremos de él".

De este modo, consideramos que la delimitación histórica del Área Metropolitana de Rosario -planteada en los distintos planes y organismos de planificación a través de la historiaresulta desactualizada; ya que, las nuevas dinámicas metropolitanas en reterritorializaciones que requieren un cambio de mirada. Por lo cual, se considera necesario alterar el punto de vista y desanclarse de los límites históricos estáticos, a fin de comprender las nuevas relaciones, procesos, imaginarios y reinvenciones presentes en el territorio. Como explica Rogerio Haesbaert (2011:79): "con un nuevo recorte o "frontera", se puede legitimar ciertas identificaciones sociales previamente existentes o, lo que es más común, al mismo tiempo crear o fortalecer otras. Como todo proceso de representación territorial es sumamente selectivo, sólo algunos espacios serán "representativos" de la(s) identidad(es) que éstos ayudan a producir o reforzar". En el período de estudio, identificamos diversas reinvenciones que conducen a nuevas reterritorializaciones producidas sobre ese "otro territorio" de enfrente.

Se plantea así, una nueva perspectiva que posibilite unir ambas orillas del Paraná en una concepción integrada de la región, más allá de los límites jurisdiccionales provinciales. Es decir, se aborda el estudio del territorio metropolitano de Rosario haciendo foco en el río Paraná como elemento estructurador y unificador entre ambas orillas. Ampliar la mirada simboliza, en este sentido, un espacio de oportunidad y de nuevas potencialidades 
para comprender las lógicas territoriales y así, producir reterritorializaciones atentas a este palimpsesto territorial -junto a la complejidad de la interacción de sus capas antiguas con las contemporáneas-.

\section{Referencias}

Ascher, F. (2007). Los nuevos principios del urbanismo. Madrid: Alianza Editorial.

Borja, J. (2012). El fin de la anticiudad posmodernista y el derecho a la ciudad en las regiones metropolitanas. En: M. BELIL, J. BORJA y M. CONTI (eds.), Ciudades, una ecuación imposible (279-320). Buenos Aires: Café de las ciudades.

Caballero, A. (Coord) (1991). Proceso de Formación urbano-territorial $y$ dinámica de transformación del Área Metropolitana de Rosario. Rosario: CFI.

Calzada, J. (2012). Argentina como productor y exportador de granos. Importancia del Gran Rosario. Informe de la Bolsa de Comercio. Bolsa de Comercio de Rosario.

Castells, M. (2013). La región metropolitana en red como forma urbana de la era de la información. Revista $\tilde{N}$ Clarín (Buenos Aires), 28 de Junio de 2013.
Ciccolella, P. (2011). Metrópolis latinoamericanas: más allá de la globalización. Quito: OLACCHI.

Ciccolella, P. (2012) Revisitando la metrópolis latinoamericana. Revista Riurb (Barcelona, Buenos Aires, Palma de Mallorca), 8, 9-21.

Corboz, A. (1983) El territorio como palimpsesto. Revista Diogéne (México), enero-marzo 1983, 121, 14-35.

Font, A. (2007). La explosión de la ciudad. Transformaciones territoriales en las regiones urbanas de la Europa Meridional. España: Ministerio de Vivienda.

Galimberti, C. (2014) La reinvención del río. Procesos de transformación de la ribera de la Región Metropolitana de Rosario, Argentina. Tesis doctoral. Directora: Dra. Arq. Isabel Martínez de San Vicente. Co-directora: Dra. Arq. Bibiana Cicutti. [Inédita].

Guattari, F.; Rolnik, S. (2006). Micropolítica. Cartografías del deseo. Madrid: Traficante de Sueños.

Haesbaert Da Costa, R. (2011). El mito de la desterritorialización: del "fin de los territorios" a la multiterritorialidad. México: Siglo XXI. 
Harvey, D. (2000). Espacios de esperanza. Madrid: Ediciones Akal S.A.

Heidegger, M. (1951). Construir, habitar, pensar En M.HEIDEGGER, J. O. GASSET, M. OLASAGASTI y E. MASSIP-BOSCH, Construir, habitar, pensar. Barcelona: ETSAB-UPC.

Indovina, F. (2007). La metropolización del territorio. En A. FONT (ed.), La explosión de la ciudad. Transformaciones territoriales en las regiones urbanas de la Europa Meridional. España: Ministerio de Vivienda.

Magnaghi, A. (2011). El proyecto local. Hacia una conciencia del lugar. Barcelona: Architectonics -UPC.

Municipalidad de Rosario (2004). Plan Estratégico Metropolitano. Región Rosario. Documento Base. (Versión Preliminar). Rosario

Oficina de Asuntos Metropolitanos (2007) Anteproyecto de Ley de Creación de Regiones Metropolitanas. Rosario.
Ortiz, R. (1998). Otro territorio: ensayos sobre el mundo contemporáneo. Colombia: Convenio Andrés Bello.

Pain, K. and Hall, P. (2006). The Polycentric Metropolis. Londres: Earthscan Publications Ltd.

PIERRI, J. (2006). El boom de la soja. Un retorno al pasado. Realidad Económica (Buenos Aires) 219, 53-63.

Reboratti, C. (2001). La Geografía entre límites, escalas y fronteras. En J. L. PALACIO-PRIETO y M. T. SÁNCHEZ SALAZAR (Eds.), Geografía para el tercer milenio. México: UNAM.

Saer, J. J. (2010). El río sin orillas: Tratado Imaginario. MadridBuenos Aires: Alianza Editorial S.A.

Soja, E. (2008). Postmetrópolis. Estudios críticos sobre las ciudades y las regiones. Madrid: Traficantes de Sueños. 